\title{
TOMMI - DEUTSCHER KINDERSOFTWAREPREIS 2020: Aufruf für Publisher, Kinderjury und Kindergärten
}

\author{
Neue Kategorie „Bildung“ - Neue Partner - \\ Neu: TOMMI Förderpreis Kindergarten
}

https://doi.org/10.1515/bd-2020-0089

Der TOMMI 2020 findet statt und wächst: Wie jedes Jahr werden die drei besten Apps, PC- und Konsolenspiele oder elektronisches Spielzeug für Kinder gesucht. Mit „Bildung“ erhält der TOMMI eine neue wichtige Kategorie, in der ebenfalls Spiele zu Lern- und Bildungsthemen ausgezeichnet werden. Hierfür wurde die Fachjury mit Bildungsexpert*innen aus Theorie und Praxis erweitert. Sobald die Pädagog^innen, Wissenschaftler*innen und Journalist*innen die diesjährigen Spiele nominiert haben, testet die TOMMI-Kinderjury die ausgewählten Titel in rund 20 Bibliotheken in ganz Deutschland.

\section{Kinderjury gesucht}

Auch dieses Jahr können sich Kinder zwischen 6 und 13 Jahren für die Kinderjury bewerben. Dafür suchen die teilnehmenden Bibliotheken aus ganz Deutschland Kinder, die Lust haben, die nominierten Spiele zu testen und zu bewerten. Kontaktdaten für die Bewerbung gibt es unter: www.kindersoftwarepreis.de/teilnah mebedingungen/\#kinderjury.

\section{Schirmherrschaft}

Der TOMMI steht unter der Schirmherrschaft der Bundesfamilienministerin Dr. Franziska Giffey. Die Verleihung ist im Rahmen der Frankfurter Buchmesse am Freitag, den 16.10.2020 geplant.

\section{Was können Publisher einreichen?}

Eingereicht werden können Games und Bildungssoftware für PC und Konsole, Apps, elektronisches Spielzeug und Webseiten. Altersgrenze ist die USK 6. Einreichungsformulare und Teilnahmebedingungen sind unter www.kindersoftwarepreis.de zu finden. Teilnahmeschluss für Publisher ist der 6. August 2020. 


\section{Neue Partner beim TOMMI und erstmalig TOMMI Förderpreis Kindergarten}

Als neue Partner des TOMMI konnten mobilsicher.de, Deutschlandfunk Kultur und die Auerbach Stiftung gewonnen werden, die den Sonderpreis „Kindergarten \& Vorschule“ gemeinsam mit Prof. Dr. Aufenanger von der Uni Mainz um den TOMMI Förderpreis Kindergarten erweitert hat. Gesucht werden Kindergärten, die innovative und praxiserprobte Konzepte zum Einsatz digitaler Medien in Kitas entwickelt haben. Der Förderpreis ist mit 1.500 Euro dotiert.

Dazu Steffen Heil, Vorstand der Auerbach Stiftung: „Der neue Preis soll Kitas in ihrer medienpädagogischen Arbeit unterstützen. Es geht darum, Kinder bereits von klein auf an eine gesunde und kreative Nutzung digitaler Medien heranzuführen.“

Teilnahmebedingungen und Einsendeschluss für den Förderpreis unter www. kindersoftwarepreis.de/kindergarten.

\section{Die Fachjury TOMMI Kindersoftwarepreis 2020}

Vorsitz: Thomas Feibel (FEIBEL.DE)

Prof. Dr. Stefan Aufenanger (Universität Mainz), Prof. Dr. Jan M. Boelmann (Zentrum für didaktische Computerspielforschung, Prof. Dr. Linda Breitlauch (Hochschule Trier), Christoph Drösser (Freier Journalist in San Francisco), Martin Eisenlauer (Bild am Sonntag), Julia Fastner (jugendschutz.net), Stephan Freundorfer (Journalist), Tim Gailus (KiKA, „Timster“), Carsten Görig (für „Der Spiegel“), Moses Grohé (love4games.org), Holger Gutwald-Rondot (Schulleiter, KraichgauRealschule Sinsheim), Steffen Haubner (Kölner Stadtanzeiger), Steffen Heil (Auerbach Stiftung), Daniel Heinz (Digitale Spiele bei der Fachstelle für Jugendmedienkultur NRW), Dr. Lisa König (Zentrum für didaktische Computerspielforschung), Martin Leupold (Schulleiter der Grundschule Wendisch Evern), Anatol Locker (Journalist), Dr. Kathrin Mertes (Projektmanagerin und Medienpädagogin Digitalpakt Schule RLP, Investitions- und Strukturbank Rheinland-Pfalz, Mainz), Matthias Rode (ZDF-Kinder- und Jugendprogramm), Tina Römer (Oberstudienrätin an der Christian-Rauch-Schule, Bad Arolsen), Kurt Sagatz (Tagesspiegel), Prof. Frauke Schade (Hochschule Angewandte Wissenschaften Hamburg), Ava Schmidle (Schülerin), Linda Scholz (Spieleratgeber-NRW), Prof. Dr. Friederike Siller (Technische Hochschule Köln), Jürgen Sleegers (Technische Hochschule Köln), Dr. Michael Spehr (Frankfurter Allgemeine Zeitung), Lukas Teutloff (Bibliothek Wolfsburg), Dorothee Wiegand (c’t).

\section{Herausgeber und Partner des Preises}

Herausgeber des TOMMI ist das Büro für Kindermedien FEIBEL.DE in Berlin. Partner des Preises sind die Auerbach Stiftung, der Deutsche Bibliotheksverband e.V. (dbv), Deutschlandfunk Kultur, Familie \& Co, die Frankfurter Buchmesse, 
Google, jugendschutz.net, mobilsicher.de, Partner \& Söhne und das ZDF-Kinderund Jugendprogramm.

\section{Diese Bibliotheken suchen eine Kinderjury.}

Bei Interesse bitte direkt Kontakt aufnehmen für Bewerbungsfrist und Bewerbungsbogen:

\begin{tabular}{ll}
\hline Baden-Württemberg & $\begin{array}{l}\text { Stadtbücherei Biberach an der Riß - Medien- und Informations- } \\
\text { zentrum }\end{array}$ \\
& $\begin{array}{l}\text { Stadtbibliothek Ludwigsburg } \\
\text { Stadtbibliothek Freiburg } \\
\text { Stadtbibliothek Pforzheim }\end{array}$ \\
& $\begin{array}{l}\text { Münchner Stadtbibliothek am Gasteig } \\
\text { Stadtbibliothek Unterschleißheim }\end{array}$ \\
Bayern & Stadtbibliothek Berlin-Lichtenberg: Anna-Seghers-Bibliothek \\
Berlin & Gemeindebibliothek Blankenfelde-Mahlow \\
Brandenburg & Bücherhallen Hamburg: Bücherhalle Alstertal \\
Hamburg & Stadtbücherei Frankfurt am Main: Bibliothekszentrum Sachsen- \\
Hessen & hausen \\
Mecklenburg-Vorpommern & Stadtbibliothek Ribnitz Damgarten \\
Niedersachsen & Stadtbibliothek Hannover \\
& Stadtbibliothek Wolfsburg \\
Nordrhein-Westfalen & Stadtbibliothek Leverkusen \\
Sachsen & Stadtbibliothek Euskirchen \\
Sachsen-Anhalt & Leipziger Städtische Bibliotheken \\
Schleswig-Holstein & Stadt- und Schulbibliothek Landsberg \\
\hline
\end{tabular}

\section{Pressekontakt:}

Thomas Feibel | FEIBEL.DE | Büro für Kindermedien Jenaer Straße 15 | 10717 Berlin Tel.: +49 (0)30 85733030 | Mobil: +49 (0)1729319446

URL: www.feibel.de, www.kindersoftwarepreis.de 